\title{
BEHANDLUNG von GERINGFÜGIGEN STREITIGKEITEN Im TÜRKISCHEN ZIVILPROZESSRECHT
}

(Small Claim Courts in Turkey)

Prof. Dr. Baki KURU

Professor für Zizilprozessrecht an der juristischen Fakultaet der Universitaet zu Ankara

1. a) In der Türkei bestehen keine besonderen Gerichte für die Erledigung geringfügiger Streitigkeiten. Geringfügige Streitigkeiten werden auch von den regulaeren (ordentlichen) Gerichten ${ }^{1}$ erledigt.

$\mathrm{Da}$ für Streitigkeiten über vermögensrechtliche $\mathrm{An}$ sprüche, deren Gegenstand an Geld oder Geldeswert die Summe von 300 USA Dollar (5000 türkische Lira) nicht übersteigt, Amtsgerichte zustaendig ${ }^{2}$ sind, werden geringfügige Streitigkeiten auch von Amtsgerichten erledigt.

b) Amtsgerichte sind mit Einzelrichtern besetzt (Amtsrichter). Alle Amtsrichter sind juristisch ausgebildete Berufsrichter.

1 Wie im westdeutsohen Recht, sind die ordentlichen Gerichte erster Instanz im türkischen Recht Amtsgerichte und Landgerichte. Da wir keine Berufungsgerichte haben (siehe unten Fussnote 3), gibt es keine Oberlandesgerichte. Der oberste Gerichtshof (zweite Instanz) ist das Kassationsgericht (siehe unten Fussnote 3).

2 Für Streitigkeiten über vermögensrechtliche Ansprüche, deren Gegenstand an Geld oder Geldeswert die Summe von 300 USA Dollar (5000 türkische Lira) übersteigt, sind Landgerichte zustaendig. In Ankara, Istanbul und Izmir sind beim Landgericht Kammern für HandeIssachen gebildet. Diese Handelskammern sind für Handelssachen zustaendig, deren Gegensund an Geld oder Geldeswert die Summe von 300 USA Dollar übersteigt. Für Handelssachen also, deren Gegenstand die Summe von 300 USA Dollar nicht übersteigt, sind in Ankara, Istanbul und Izmir auch Amtsgerichte zustaendig. 
c) Die Zustaendigkeit der Amtsgerichte (und Landgerichte) ist zwingend (ausschliesslich). Die Gerichte müssen ihre eigene Zustaendigkeit von Amtes wegen prüfen.

d) Im Rahmen des Konsumentenschutzes bestehen in der Türkei keine Sondergeriohte, deren Aufgabe es ist, vornehmlich geringfügige Fragen des Konsumentenschutzes zu entscheiden.

2. a) $\mathrm{Da}$ in der Türkei dafür keine besonderen Gerichte bestehen, kann mit Sicherheit nicht gesagt werden, welche Streitigkeiten in der Türkei als "geringfügige Streitigkeiten" gelten. Man kann aber Streitigkeiten über Geldforderungen und Ansprüche an beweglichen Sachen, deren Gegenstand die Summe von 120 USA Dollar (2000 türkische Lira) nicht übersteigt, als "geringfugige Streitigkeiten" bezeichnen. Denn gegen Urteile, durch die über Geldforderungs-und Fahrnisklagen entschieden wird, deren Streitgegenstand die Summe von 120 USA Dollar nicht übersteigt, ist kein Rechtsmittel (also keine Revision) zulaessig?. Die Revisionssumme betraegt also 120 Dollar4.

b) Wie bereits erwaehnt, richtet sich die Abgrenzung nach einem bestimmten Geldwert des Streitgegenstandes (120 USA Dollar).

c) Es bestehen Ausnahmen, die ohne Rücksicht auf den geringen Wert nicht der Zustaendigkeit der Amtsgerichte, sondern der Zustaendigkeit der Landgerichte gehören. Zum Beispiel Streitigkeiten betreffend Stiftungen und Urheberrecht ${ }^{\mathrm{s}}$. Sie werden zwar hinsichtlich der Revisions-

${ }^{3}$ Das tïrkische Zivilprozessrecht kennt nur ein Rechtsmittel; das ist die Revision. Es gibt also keine Berufungsgerichte und keine Berufung. Gegen Urteile der Amtsgerichte und der Landgerichte, duroh die über Klagen entschieden wird, deren Streitgegenstand die Summe von 120 USA Dollar ïbersteigt, ist nur das einzige Rechtsmittel "Revision* zulaessig. Es gibt nur ein Revisionsgericht in Ankara (das türkische Kassationsgericht). Das Kassationsgericht hat 15 Zivilkammern und 9 Strafkammern (vgl. oben Fussnote 1).

${ }^{4}$ Es ist zu bemerken, dass die Revisionssumme nícht nach dem Beschwer. degegenstand, sondern nach dem Streitgegenstand der Klage berechnet wird.

${ }^{5}$ Alle Mietstreitigkeiten gehören zur Zustaendigkeit de. Amtsgerichte; und zwar ohne Rücksicht auf den Wert des Streitgegenstandes. 
summe wie andere (der Zustaendigkeit der Amtsgerichte gehörende) geringfügige Streitigkeiten behandelt. Sie werden aber nicht nach dem (bei Amtsgerichten anzuwendenden) summarischen Verfahren, sondern nach dem (bei Landgerichten anzuwendenden) ordentlichen Verfahren erledigt.

d) Die Regeln für geringfügige Streitigkeiten gelten auch für Streitigkeiten aus Arbeits-und Dienstverhaeltnissen, weil die Urteile der Arbeitsgerichte auch derselben Revisionsgrenze (120 USA Dollar) unterliegen und das bei Arbeitsgerichten anzuwendende mündliche Verfahren dem (bei Amtsgerichten anzuwendenden) summarischen Verfahren sehr aehnlich gestaltet ist.

3. a) Wie bereits erwaehnt, besteht für diese geringfügige Streitigkeiten kein besonderes Verfahren. Aber, da das summarische Verfahren, das bei Amstgerichten angewandt wird, eine besondere Verfahrensart ist, kann daraus gefolgert werden, dass für geringfügige Streitigkeiten auch ein besonderes Verfahren (summarisches Verfahren) angewandt wird.

b) Bis zum Jahre 1973 wurde bei Amtsgerichten mündliches Verfahren angewandt. Mit der Aenderung des türkischen Zivilprozessgesetzes im Jahre 1973 sind Amtsgerichte dem (bereits für einige Streitigkeiten bestehenden) summarischen Verfahren unterworfen. Mit derselben Gesetzesaenderung wurde auch die Revisionsgrenze von 3 USA Dollar (50 türkische Lira) auf 120 USA Dollar (2000 türkische Lira) erhöht.

c) Die Eigenarten und Abweichungen dieses summarischen Verfahrens gegenüber dem normalen (ordentlichen) Verfahren bei Landgerichten bestehen in folgenden Punkten:

aa) Es gibt im türkischen Recht keinen Anwaltszwang; auch nicht bei Landgerichten und beim Kassationsgericht. Daher müssen die Parteien nicht von Rechtsanwaelten vertreten sein. Sie können sich aber von Rechtsanwaelten (und zwar nur von ihnen) vertreten lassen'.

bb) Falls sich die Parteien eines Rechtsanwaltes bedienen, werden der obsiegenden Partei (mit den übrigen

6 Vgl. unten 5, b, ee (Seite 10). 
Gerichtskosten) auch die Kosten des Rechtsanwaltes? ersetzt.

cc) Die Einleitung des summarischen Verfahrens ist schriftlich; sein Fortgang (Verhandlung und Urteilsverküindung) ist mündlich. Die Klageerhebung beginnt mit der einreichung der Klageschrift. Der Klaeger soll seine schriftliche Beweismittel mit der Klageschrift dem Gericht ïbergeben.

Es findet keinen Schriftsatzwechsel statt. Die Parteien können ihre Antraege und Erklaerungen bei der ersten mündlichen VerhandIung zu Protokoll des Amtsgerichts abgeben.

Die Parteien werden zur ersten mündlichen Verhandlung geladen. Gegen die nicht erschienene Partei wird aber kein Versaeumnisurteil erlassen und zugestellt. Bei Saeumnis einer Partei wird also die Verhandlung weitergeführt ${ }^{8}$.

Waehrend der Gerichtsferien" werden im summarischen Verfahren Termine abgehalten und Enstcheidungen erlassen.

Es gibt kein besonderes schriftliches Verfahren oder Urkundsverfahren für geringfügige Streitigkeiten. Es gibt aber ein einfaches Vollstreckungsverfahren, das besonders für geringfügige Streitigkeiten meistens das Urteilsverfahren (den Prozessweg) erspart. Daher möchte ich hier dieses (dem deutschen und österreichischen Mahnverfahren aehnliche) einfache Vollstreckungsverfahren (Schuldbetreibung) kurz skizzieren:

Die Schuldbetreibung ${ }^{10}$ ist nur für Geldforderungen ${ }^{13}$

7 Die Anwaltsgebühren bestimmen sich nach den Vorschriften fiur das gewöhnliche Verfahren; es sind zehn Prozent der geklagten bzw. geurteilten Summe.

${ }^{8}$ Vgl. unten Seite 6 Fussnote 13.

9 Die Gerichtsferien beginnen am 20. Juli und enden am 5. September.

${ }^{10}$ Die Vorschriften über Schuldbtreibung sind nach dem Vorbild des schweizerischen Bundesgesetz tiber. Schuldbetreibung und Konkurs aufgebaut (siehe dazu: Baki Kuru, das schweizerische Schuldbetreibungs-und Konkursgesetz in der Türkei-Zeitschrift für Schweizerisches Recht, 1964, Band 83, S. 331-350; derselbe, die neue Revision des 
und Sicherheitsleistungen zulaessig. Statt für seine Geldforderung eine Klage zu erheben, kann der Glaeubiger unmittelbar beim Betreibungsamt (Zwangsvollstreckungsamt) die Schuldbetreibung verlangen, auch dann, wenn er für seine Geldforderung keine Forderungsurkunde hat. Nach Empfang des Betreibungsbegehrens erlaesst das Betreibungsamt einen Zahlungsbefehl an den Schuldner.

Erhebt der Schuldner binnen sieben Tagen keinen Rechtsvorschlag (Widerspruch) gegen den Zahlungsbefehl und zahlt er binnen derselben sieben Tagen die Forderung (samt Betreibungskosten) nicht, so kann der Glaeubiger das Pfaendungsbegehren beim Betreigungsamt stellen. Darauf werden die Sachen des Schuldners (durch das Betreibungsamt) gepfaendet, werwertet und aus dem Erlös wird der Glaeubiger befriedigt.

Erhebt der Schuldner gegen den Zahlungsbefehl Rechtsvorschlag, so wird die Schuldbetreibung eingestellt. Für die Beseitigung des Rechtsvorschlages (also für die Fortsetzung der Schuldbetreibung) stehen dem Glaeubiger nach seiner Wahl zwei Möglichkeiten offen : a) der ordentliche Prozessweg, b) die Rechtsöffnung.

Beruht die Forderung auf einer Urkunde ${ }^{12}$, so kann der Glaeubiger beim Vollstreckungsrichter die Rechtsöffnung verlangen. Er ist aber nicht gezwungen, die Rechtsöffnung zu verlangen; er kann auch den ordentlichen Prozessweg waehlen. Wenn er aber keine Forderungsurkunde besitzt, so hat er nur die Möglichkeit, den ordentlichen Prozessweg zu bestreiten.

Das Rechtsöffnungsverfahren beim Vollstreckungsrichter ist sehr einfach. Wenn der Schuldner vor dem Volistreckungsrichter durch eine Urkunde nicht beweisen kann, dass die Forderung getilgt oder gestundet

türkischen Schuldbetreibungs-und Konkursgesetz -Blaetter für Schuldbetreibung und Konkurs, 1967/2, S. 33-45-).

11 Es gibt dafür keine Höchstgrenze. Für alle Geldforderungen ist die Schuldbetreibung zulaessig.

${ }^{12}$ Diese Urkunde kann öffentliche Urkunde, durch Unterschrift bekraeftigte Privaturkunde, Wechsel oder Check sein. 
worden ist oder die Forderung verjaehrt ist, so gewaehrt der Vollstreckungsrichter die Rechtsöffnung. Darauf kann der Glaeubiger das Pfaendungsbegehren stellen. Dann werden die Sachen des Schuldners (durch das Betreibungsamt) gepfaendet, verwertet und aus dem Erlös wird der Glaeubiger befriedigt.

Dieser Schuldbetreibung kommt in der Praxis cine erhebliche Bedeutung zu. Denn viele geringfügige (auch grosse) Streitigkeiten werden dadurch erledigt und für sie wird das Urteilsverfahren (der ordentliche Prozessweg) erspart.

dd) Bei Amtsgerichten wird das Urteil in dem Termin verkündet, in dem die mündliche Verhandlung geschlossen wird $^{1 / 3}$. Das Urteil muss mit Gründen versehen $\operatorname{sein}^{14}$.

ee) Im summarischen Verfahren haben nicht juristisch ausgebildete Personen keine richterlichen Aufgaben. Alle Amtsrichter sind juristisch ausgebildete Berufsrichter.

Bei der Schuldbetreibung dagegen haben nicht juristisch ausgebildete Personen wichtige Aufgaben. Denn die Schuldbetreibung beginnt und endet beim Betreibungsamt. Das Betreibungsamt empfaengt das Betreibungsbegehren, erlaesst den Zahlungsbefehl, pfaendet und verwertet die Sachen des Schuldners und befriedigt aus dem Erlös den Glaeubiger. Wenn der Schuldner keinen Rechtsvorschlag gegen den Zahlungsbefehl erhebt, so kann das Betreibungsamt ohne richterliche Hilfe die Schuldbetreibung bis zum Ende fortsetzen. Im Falle des Rechtsvorschlages kann das Betreibungsamt die Sohuldbetreibung erst nach der Rechtsöffnung durch den Vollstreckungsrichter fortsetzen. Der Betreibungsbeamter, der das Betreibungsamt leitet, sein Stellvertreter und Angestellte des Betrei-

13 War eine Partei bei der Urteilsvenkuindung nicht anwesend, so wird ihr der Urteilstenor (Urteilsspruch) zugestellt.

14 Obwohl das Urteil in geringfiigigen Streitigkeiten (bis 120 USA Dollar) nicht angefuchten werden kann (siehe oben 2, a Seite 2), muss das Urteil (wie andere revisionsfaehige Urteile) mit Grïnden versehen sein. 
bungsamtes können zwar juristisch ausgebildet sein. Sie sind aber keine Berufsrichter, sondern Verwaltungsbeamte bzw. Angestellte. Der Vollstreckungsrichter ist dagegen juristisch ausgebildeter Berufsrichter.

ff) Wie bereits erwaehnt, ist gegen Urteile, durch die uiber Geldforderungs-und Fahrnisklagen entschieden wird, deren Streitgegenstand die Summe von 120 USA Dollar nicht übersteigt, kein Rechtsmittel (keine Revision) zulaessig ${ }^{15}$. Von dieser Ausschliessung des Rechtsmittels (der Revision) macht das Gesetz keine Ausnahme. Zum Beispiel wenn die sachliche Zustaendigkeit oder der ordentliche Rechtsweg nicht gegeben war, ist das Urteil des Amtsgerichts (oder des Landgerichts) trotzdem rechtskraeftig (unanfechtbar).

Zwar kann der Generalstaatsanwalt gegen diese (von Rechts wegen) rechtskraeftige Urteile wegen Gesetzesverletzung beim Kassationsgericht Revision einlegen und darauf kann das Urteil kassient werden. Diese Kassation hat aber auf die Rechtstellung der Parteien keine Wirkung; trotz der Kassation bleibt das Urteil gültig. Diese Kassation des Urteils wird im Staatsblatt veröffentlicht, damit alle Gerichte davon Kenntnis nehmen können und den gleichen Fehler nicht machen sollen.

Gegen diese rechtskraeftige Urteile (also bis 120 Dollar) ist die Wiederaufnahme des Verfahrens (bei demselben Amtsgericht) zulaessig. Gegen das Urteil im Wiederaufnahmeverfahren ist ebenso kein Rechtsmittel (keine Revision) zulaessig.

4. a) Die durchschnittliche Dauer des Verfahrens bei Amtsgerichten für geringfügige Streitigkeiten (bis 120 Dollar) ist schaetzungsweise fünf Monate. Uber die Anzahl dieser Streitigkeiten in den letzten Jahren kann ich nichts angeben, da es dafür keine Statistik gibt.

b) Die durchschnittliche Kosten eines solchen Verfahrens sind etwa 15 USA Dollar (250 türkische Lira); Davon sind 12 Dollar Anwaltsgebühren bzw. Sachverstaendigenkosten.

is Siehe oben 2, a (Seite 2). 
c) In dem türkischen Zivilprozessgesetz ist das Armenrecht für alle Verfahrensarten allgemein geregelt. Danach kann auch für geringfügige Streitigkeiten das Armenrecht bewilligt werden. Eine Besserstellung für geringfügige Streitigkeiten gibt es nicht ${ }^{16}$. In diesem Zusammenhang ist zu erwaehnen, dass Klagen, deren Streitgegenstand die Summe von 6 USA Dollar (100 Lira) nicht übersteigt, gebührenfrei $\operatorname{sind}^{17}$.

5. a) Zur Verbesserung und Vereinfachung des Verfahrens für geringfügige Streitigkeiten bestehen zur Zeit keine Reformprojekte.

b) Zur Vereinfachung, Verbilligung, Rationalisierung und Vermenschlichung des Verfahrens in geringfügigen Streitig. keiten habe ich persönlich folgende Vorschlaege und Anrae. gungen :

Zunaechst möchte ich bemerken, dass meiner Meinung nach für die Behandlung der geringfügigen Streitigkeiten kein Sonderverfahren (zum Beispiel Schiedsurteilsverfahren oder Bagatellverfahren) geschaffen werden soll. Denn die Parteien in geringfügiger Streitigkeiten müssen auch Anspruch auf ein ebenso gründliches Verfahren haben wie in Streitigkeiten mit höherem Streitwert. Aber zur Vereinfachung, Verbilligung, Rationalisierung und Vermenschlichung des Verfahrens für geringfügige Streitigkeiten müssen wir einige Sondervorschriften haben. Diese Sondervorschriften sollen eine weitere Vereinfachung des ohnedies einfach gestalteten summarischen Verfahrens bewirken. Naemlich:

aa) Es muss im Gesetz ausdrücklich erwaehnt werden, dass die Grenze für geringfügige Streitigkeiten (Bagatellgrenze) 120 USA Dollar (2000 türkische Lira) ist ${ }^{13}$.

bb) Alle Klagen über geringfügige Streitigkeiten (bis 120 Dollar) sollen gebührenfrei sein ${ }^{19}$.

cc) Im Verfahren wegen geringfügiger Streitigkeiten ist die Armut der Parteien in den meisten Faellen gegeben

16 Vgl. unten 5, b, cc (Seite 9).

17 Vgl, unten 5, b, bb (Seite 9).

13 Siehe oben Seite 2.

19 Vgl. oben 4, c (Seite 8). 
(vermutet), Daher muss für die Bewilligung des Armenrechts für geringfügige Streitigkeiten von der Voraussetzung der Armut abgesehen werden. Von dem Antragsteller soll also nicht verlangt werden, glaubhaft zu machen, dass er ausserstande ist, ohne Beeintraechtigung des für ihn und seine Familie notwendigen Unterhalts die Kosten des Prozesses zu bestreiten.

dd) Für geringfügige Streitigkeiten soll der Zeugenbeweis allgemein und ohne Einschraenkung zugelassen werden. Wie im französischen ${ }^{20}$ und italienisohen ${ }^{\text {th }}$ Recht, ist im türkischen Zivilprozessrecht der Zeugenbeweis bei Rechtsgeschaeften mit einem bestimmten Mindestgeschaeftswert ausgeschlossen. Nach Art. 288 des türkischen Zivilprozessgesetzes ist der Zeugenbeweis ausgeschlossen bei allen Rechtsgeschaeften, deren Gegenstand die Summe von 30 USA Dollar (500 tirkische Lira) ibersteigt ${ }^{n}$. Da Rechtsgeschaefte mit geringfiigigen Geschaeftswert meistens nicht beurkundet zu werden pflegen, soll diese Summe (von 30 Dollar) auf 120 Dollar (2000 türkische Lira) erhöht werden.

ee) Wie oben erwaehnt ${ }^{23}$, gibt es im tiirkischen Recht keinen Anwaltszwang. Wenn aber eine Partei die Klage durch einen Bevollmaechtigten führen will, so kann sie sich nur durch einen Rechtsanwalt vertreten lassen. Da aber für geringfügige Streitigkeiten einer Partei kaum möglich ist, einen Rechtsanwalt zu finden, der sich mit der geringfügigen Gebühr (gemaess der Rechtsanwaltsgebührenordnung) begniigen wird, soll den Parteien die Möglichkeit gegeben werden, ihre Klagen iber geringfügige Streitigkeiten durch jede prozessfaehige Person als Bevollmaechtigten zu führen.

20 Siehe Code Civil Art. 1341.

21 Siehe Codice Civile Art. 2721.

2 Der innere Grund für diese Regelung ist die Unzuverbaessigkeit des Zeugen als Beweismittel. Danach können also für Rechtsgeschaefte, deren Gegenstand die Summe von 30 USA Dollar übersteigt nur die Urkunde als Beweismittel herangezogen werden. Durch Art. 288 wird aber die Gültigkeit eines nicht urkundlich abgeschlossenen Rechtsgeschaefts nicht beeintraechtigt. Es wird ledigdich der Zeugenbeweis ausgeschlossen. Zulaessig bleiben (ausser der Urkunde) das Gestaendnis und der Parteieid. 3 Siehe aben Seite 3. 
ff) Da wegen so eben gesagten Gründen die Parteien für geringfügige Streitigkeiten in der Regel nicht duroh Rechtsanwaelte vertreten sein werden, soll das Gericht den Parteien eine gewisse Fürsorge gewaehren (behilflich sein). Naemlich :

1 - Der Richter soll in jeder Lage des Verfahrens auf die gütliche Bëlegung des Rechtsstreites, d.h. auf den Abschluss eines Prozessvergleichs hinwirken.

2 - Das Gericht soll von sich aus Beweismittel heranziehen und von den Förmlichkeiten des Beweisrechts abweichen können.

3 - Afle Zustellungen sollen von Amtes wegen erfolgen.

c) Eine Ubertragung der Entscheidung geringfügiger Streitigkeiten an nicht juristische Einrichtungen ist nach der tiirkischen Verfassung nicht möglich. Denn nach Art. 7 der türkischen Verfassung wird die Rechtssprechungsfunktion von unabhaengigen Gerichten wahrgenommen. Diese Vorschrift wird so ausgelegt, dass die Gerischtsbarkeit ausschliesslich von unabhaengigen Gerichten wahrgenommen werden kann. Eine Ubertragung auf andere Stellen oder Personen ist ausgeschlossen (Art. 4 Abs. 3 der türkischen Verfassung ${ }^{24}$. Ausserdem halte ich eine Ubertragung der Entscheidung geringfügiger Streitigkeiten an nicht juristische Einrichtungen nicht für sinnvoll, weil die Parteien in geringfügiger Streitigkeiten auch Anspruch auf ein ebenso grïndliches Verfahren haben müssen wie in Streitigkeiten mit höherem Streitwert.

2* Die Schuldbetreibung (Siehe oben Seite 56 und 6-7) ist keine Gerichto barkeit. Dagegen ist die Rechtsöffnung (siehe oben Seite 7-8 und 9) eine Rechtssprechungsfunktion. Daher ist für die Rechtsöffnung ein unab. haengiger Berufsrichter (Vollstreckungsrichter) ausschliesslich zustaendig. 


\section{(Summary in English) \\ SMALL CLAIM COURTS IN TURKEY}

Prof. Dr. Baki KuRU (Ankara)

There are no special courts for small claims in Turkey. Small claims handled by general courts are within the jurisdiction of civil courts of peace, since these courts may settle claims of up to 300 US Dollars (5000 Turkish Liras).

A civil court of peace has a single judge.

Since there are no special courts for small claims it is impossible to determine which cases are considered "small claims*. However, we might qualify cases of up to 120 US Dollars (2000 Turkish Liras) as small claims. Because court decisions ending such cases cannot be appealed at any level; that is to say they are final.

"Simplified procedure» is followed in civil courts of peace. So small claims are subject to this type of procedure. In simplified procedure a lawsuit is started by a written petition. But the trial and the pronouncement of the judgement are done orally. During the first session the parties tell the judge their claims and defences, and the judge has them recorded in the minutes. If one of the parties do not attend the trial there is no need for a formal "decision of default"; the trial continues in the absence of one of the parties. These cases continue during the annual judicial recess.

Such lawsuits dealing with small claims last an average of 5 months. And the court costs are an average of 15 US Dollars (250 Turkish Liras).

Following are my proposals for a simpler, faster, cheaper and more human procedure for small claims :

In small claims (cases of up to 120 US Dollars) the parties should be exempt from paying judicial duties.

In such lawsuits if one of the parties requests legal aid he must not carry the burden of proving that he is unable to pay necessary expenses.

Proof by witnesses should be allowed in these lawsuits. Under article 288 of the Turkish Code of Civil Procedure any legal trans- 
action whose value exceeds 30 US Dollars (500 Turkish Liras) must be proved by written evidence (deed). This minimum amount should be raised to 120 US Dollars (2000 Turkish Liras).

In these lawsuits the parties must be able to assign anyone as their representatives, not only lawyers.

The court must be more helpful to the parties in small claims: The court must encourage the parties to end the dispute by peaceful settlement. The court must be able to request an its own from the parties to show additional evidence. Service to the parties must be done automatically and not upon the request of the parties. 